\title{
Multiscale Relative Transition Entropy Analysis of Electroencephalogram
}

\author{
Ying Wang ${ }^{1}$, Fengzhen $\mathrm{Hou}^{2}$, Jiafei Dai, a , Jin $\mathrm{Li}^{4}$, Jun Wang ${ }^{1, \mathrm{~b}}$ \\ ${ }^{1}$ Image Processing and Image Communications Key Lab., Nanjing Univ. of Posts \& Telecomm., \\ Nanjing, 210003, China \\ ${ }^{2}$ School of Science, China Pharmaceutical University, Nanjing, 210009, China \\ ${ }^{3}$ Nanjing General Hospital of Nanjing Military Command, Nanjing, 210002, China \\ ${ }^{4}$ College of Physics and Information Technology, Shaanxi Normal University - Xi'an, 710062, China

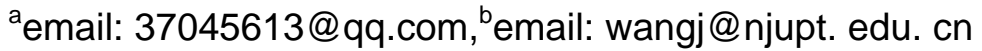

Keywords: multiscale, relative transition entropy, complexity.

\begin{abstract}
Recently, quantifying the complexity of the physiological time series has become more and more concerned. As one of the most complex physiological signals, electroencephalogram (EEG) including a large number of physiological and pathological information attracts widespread interest. However, many traditional algorithms fail to account for the multiple times scales inherent in physiologic dynamics. In this paper, we proposed multiscale relative transition entropy algorithm (MRTE) to analyze the white noise and pink noise, the adolescent and adults EEG as well as normal and epileptic EEG. The results indicate that there are distinct tendency among different types EEG which indicating that the multiscale relative transition entropy can distinguish different physiological and pathological signals.
\end{abstract}

\section{Introduction}

The EEG signal is generated by the activities of brain and is a spontaneous electrical activity always existed in the central nervous systems, which is an important bioelectric signal[1]. In clinical diagnosis, as EEG contains a large number of physiological and pathological information, the deeply research on it can bring lots of benefits: the reliability and accuracy of diagnosis and detection in the brain lesions will be improved. At the same time, the research will also provide an effective means of the diagnosis among brain diseases.

Caused by any neurological disease of the brain, such as cerebrovascular disease, migraine, epileptic and so on, changes in terms of brain structure and function can lead to the abnormalities of EEG. As a result, the analysis processing and feature extraction of EEG will be important in the identification of brain disease, the prediction and prevention of pathological states.

Entropy is a parameter to measure the chaos degree of system with a more special definition in different fields and in life sciences, it means disorder life activities. In order to get a comprehensive study of complexity of EEG, this paper adopts a toll, called relative transition entropy ( $r H$ )[2-3], akin to the symbolic transfer entropy which integrated the concept of transfer entropy and relative entropy[4-6]. This algorithm calculates the relative entropy by the transfer probabilities between the forward sequence and the reverse sequence which leads to obtain the degree of irreversible process[7-8].

\section{Theory of multiscale relative transition entropy}

The purpose of this paper is to apply a general approach to take into account the multiple time scales[9-11] in EEG signals with the relative transition entropy. In different scales, the paper showed the distinction of the relative transition entropy among different types of EEG signals. At the same time, the appropriate scale can be more beneficial to the analysis of illness which can play a positive and important role to further improve the effect of the clinical diagnosis. We study 
simulated noises as well as human EEG signals, the latter representing the output of an important physiologic system.

Giving a one-dimensional discrete time series, $\{x(i): 1 \leq i \leq N\}$, for a fixed scale factor $\tau$, we construct consecutive coarse-gained time series, $\left\{y^{(\tau)}\right\}$, with the equation,

$$
y_{j}^{(r)}=\frac{1}{r} \sum_{(j-1) r+1}^{j r} x_{i},\left(1 \leq j \leq \frac{N}{r}\right)
$$

The length of each coarse-gained time series depends on the scale factor, $\tau$. Here we consider time series with 5040 points and coarse-grain temp up to scale 10, so that the shortest time series has 504 points. Then, for each coarse-gained time series, calculating a relative transition entropy as a function of the scale factor $\tau$.

Regarding the new coarse sequences as participants, we map the sequences into symbolic strings. For original time series, the symbolic map[12-13] can be introduced as follows:

$$
s_{i}(\underset{I}{\&})=\left\{\begin{array}{llll}
0: & \mu_{1}<x_{i} \leq(1+a) u_{1} & \text { or } & (1+a) \mu_{2} \leq x_{i}<\mu_{2} \\
1: & (1+a) \mu_{1}<x_{i}<\infty & \text { or } & -\infty<x_{i}<(1+a) \mu_{2} \\
2: & (1-a) \mu_{1} \leq x_{i}<\mu_{1} & \text { or } & \mu_{2}<x_{i} \leq(1-a) \mu_{2} \\
3: & (1-a) \mu_{2}<x_{i}<(1-a) \mu_{1} &
\end{array}\right.
$$

Where $\mathrm{i}$ is the index of series, with $\mathrm{i}=1,2, \ldots, \mathrm{N}$. The time series $x_{1}, x_{2}, x_{3}, \ldots, x_{N}$ is transformed into the symbol sequence $s_{1}, s_{2}, s_{3}, \ldots, s_{N}, s_{i} \in \mathrm{A}$ on the basis of the alphabet $\mathrm{A}=\{0,1,2,3\}$. The transformation into symbols refers to three given levels where $\mu_{1}$ denotes the mean of all series that greater than zero while $\mu_{2}$ denotes the mean of all series that less than zero. The parameter $\alpha$ [14] is a special parameter that we have chosen 0.05 . Symbolization is a coarse-grained process. Some detailed information is lost in this process, but the coarse dynamic behavior can be analyzed.

For the new symbol sequence, $r H$ [2]needs to be calculated:

$$
r H_{n \| m}=\sum_{X, Y} p\left(x_{t-n}^{t}, y_{t-m}^{t}\right) \bullet D\left(\left(P\left(x_{t} \mid x_{t-n}^{t-1}\right) \|\left(p\left(y_{t} \mid y_{t-m}^{t-1}\right)\right)\right.\right.
$$

Where $x_{t-n}^{t}=x_{t-n}, x_{t-n+1}, \ldots, x_{t}, y_{t-m}^{t}=y_{t-n}, y_{t-n+1}, \ldots, y_{t}, x_{t-n}^{t}$ refers to the forward sequence while $y_{t-m}^{t}$ are the reverse sequence. Change the scales can get different $r H$.

\section{Results}

With the above algorithms, we proceed to analyze the advantage of the multiscale relative transition entropy compared to the conventional method for the EEG signals, white noise and pink noise. There are two types of the EEG signals.One of them is from adolescence and adult, the other is EEG from normal subjects and epilepsy patients. Both of the data are recorded at Nanjing General Hospital of Nanjing Military Command.For these two kinds of signals, the length of each one is over one minute and the sampling frequency is $512 \mathrm{~Hz}$ as well as contains 16 lead signals. As c3 signal has better discrimination in scale 1, so we chose c3 lead signals as research object.

We first test this method on simulated white and pink noises. The relationship between scale factor and entropy of these noises, is, respectively, plotted in fig. 1. As shown in fig. 1, for every scale, the relative transition entropy of pink noises is higher than the white noises. However, while the entropy value for the coarse-gained 1/f series slowly decreases for all scales, the entropy value for the coarse-gained white noise time series ascends sluggishly. This result is consistent with the fact that, unlike white noise, $1 / \mathrm{f}$ noise is closer to the body's physiological signals with higher complexity. Actually, an entropy value of a signal is generally higher than the other among the most scale, indicating it's higher complexity. 


\section{the relationship between scale factor and entror}

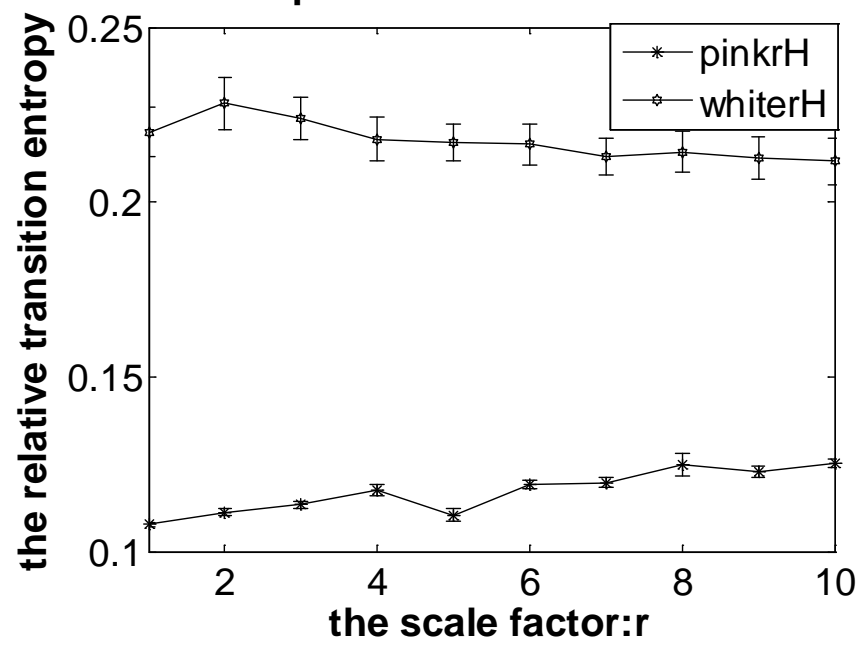

Fig.1: The analysis of Gaussian distributed white noise and 1/f noise. The total length of the original series is 5040 points.

In order to further analyze the properties of the multiply scales for relative transition entropy, we applied the method to the selected physiologic time series (Fig. 2). The overall trend of the two signals is consistent and they reach the maximum at the scale 2 and the scale 3 . When $r<3$, the entropy of the adult is higher than the adolescent, which indicating the adult EEG shows higher activity. For among the data in this experiment, the average age of adult objects is about 35 while the adolescent is about 18, so the adult brain is in the most active period. While it becomes contrary as $r>3$, which states that adolescents EEG shows higher activity, and both complexity are reduced. If scale in multiscale process is bigger, the obtained sequences more reflect the low frequency components in the signal. the EEG often include $\delta$ wave whose frequency is between 1 and $3.5 \mathrm{~Hz} 、 \theta$ wave whose frequency is between 4 and $7 \mathrm{~Hz}$ 、 $\alpha$ wave whose frequency is between 8 and $13 \mathrm{~Hz} 、 \beta$ wave whose frequency is between 13and $30 \mathrm{~Hz}$.Somebody who is in infancy or intellectual development is not mature and $\delta$ wave is at higher weights, while $\alpha$ wave is the basic rhythms of EEG of normal adults. The larger scale means the lower frequency with the higher probability of $\delta$ wave. So the adolescents show higher relative transition entropy than the adults in large scale.

the relationship between scale factor and entror

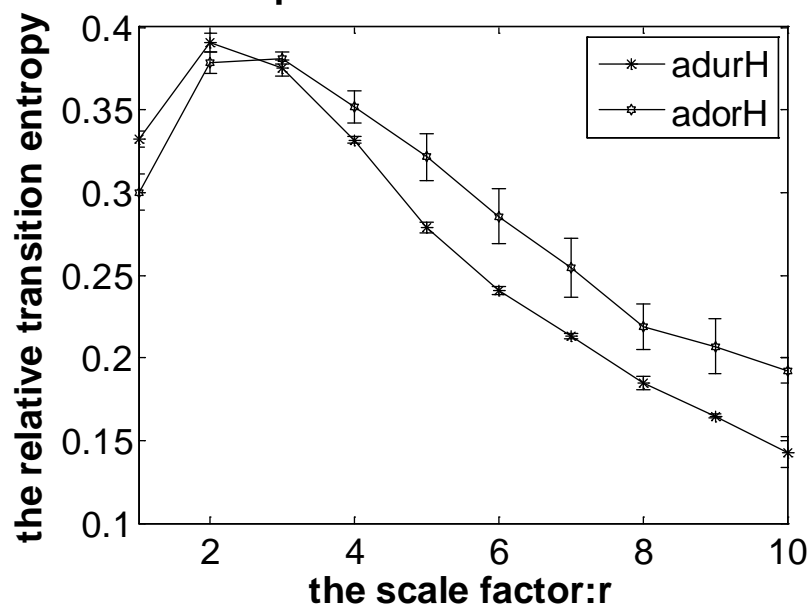

Fig.2: the associated sequences are eight subjects: four adolescent subjects and four adult subjects. The total length of the original series is 5040 points. adurH--->adult $\mathrm{rH}$, adorH--->adolescence rH.

Last, we proceed to compare the relative transition entropies of the heathy subjects with the patients. From the Fig.3 we can draw a conclusion that for the scale one, the relative transition entropies of the healthy subjects are significantly higher than the patients (by t test, $\mathrm{T}$ value $=2.361$, $\mathrm{P}=0.03$, as under the $\mathrm{P}<0.05$ level). The relative transition entropy value is lower means 
self-similarity of the sequence is higher and the sequence is more simple. With the increase of the scale, r, two kinds of the entropy are tended to first increased and then decreased, with respect to the scale one, the discrimination has been greatly improved. For normal subjects, $\alpha$ waveform is at bigger weights, while $\delta$ and $\theta$ waves are at bigger weights of the epilepsy. The larger scale indicates the lower resampling frequency. The $\delta$ and $\theta$ waves frequency are lower than the $\alpha$ wave, so the epilepsy patients show higher relative transition entropy than the normal subjects in large scale. When $\mathrm{r}$ is between 2 and 3, the two kinds subjects' entropy values are almost equal. At this moment, it is obvious that the two signals can not be distinguished. So there are apparent advantages of multiscale process, it can greatly improve the discrimination among the different EEG signals but choose the adaptive scale is more important.

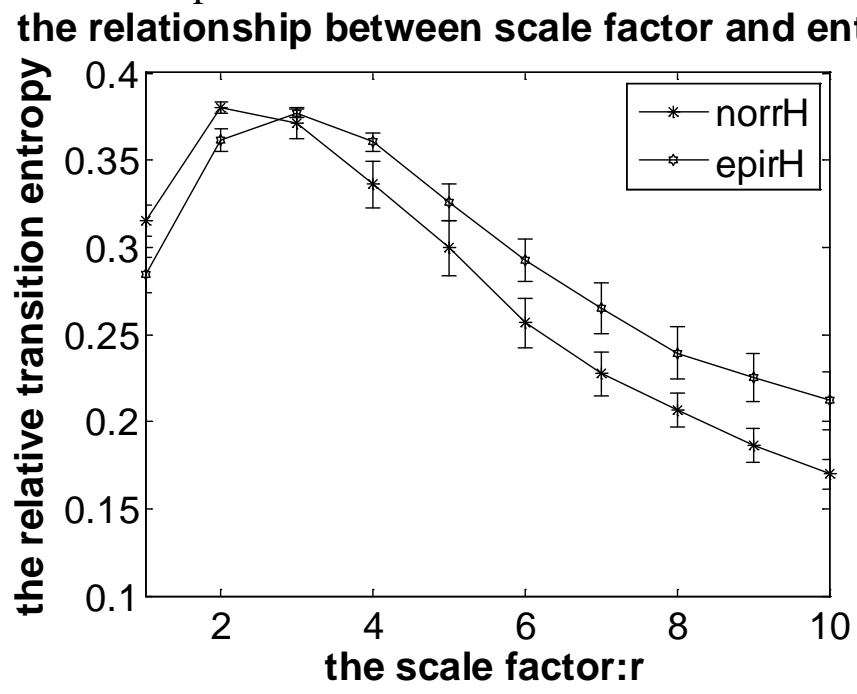

Fig.3: the associated sequences are twenty subjects: ten normal subjects and ten epileptic subjects.

The total length of the original series is 5040 points.

\section{Conclusion}

In order to obtain better dynamic characteristics, we present the multiscale method based on the relative transition entropy. Complex dynamics typically reveal structure on multiple spatial and temporal scales, these multiscale features, ignored by conventional entropy methods, are explicitly addressed in the multiscale algorithm. For different signals, as the scale changes, the entropy will change too. From the above experiments, we know that applying this new method to the clinical medical detection has an important significance and this method can also be used to apply to other areas.

\section{Acknowledgements}

In this paper, the Project supported by the National Natural Science Foundation of China (Grant Nos. 61271082,61201029,61102094,61401518), the Natural Science Foundation of Jiangsu Province (Grant Nos.BK20141432) , the Foundation of Nanjing General Hospital of Nanjing Military Command (2014019) and the Fundamental Research Funds for the Central Universities (FY2014LX0039).

\section{References}

[1] Zhang, M., Cui, C., Ma, Q. L., Gan, Z. L. \& Wang, J., Coupling analysis of multivariate bioelectricity signal based symbolic partial mutual information. Acta Physica Sinica, 62(6),pp. 068704, 2013.

[2] Wang, Y., Hou, F.-Z., Dai, J.-F., Liu, X.-F., Li, J. \& Wang, J., Analysis on relative transfer of entropy based on improved epileptic EEG. Acta Physica Sinica, 63(21),pp. 218701, 2014. 
[3] Jones, N. S., Using the memories of multiscale machines to characterize complex systems. Physical Review Letters, 100(20),pp. 208702, 2008.

[4] Schreiber, T., Measuring information transfer. Physical Review Letters, 85(2),pp. 461-464, 2000.

[5] Staniek, M. \& Lehnertz, K., Symbolic transfer entropy. Physical Review Letters, 100(15),pp. 158101, 2008.

[6] Andrieux, D., Gaspard, P., Ciliberto, S., Garnier, N., Joubaud, S. \& Petrosyan, A., Entropy Production and Time Asymmetry in Nonequilibrium Fluctuations. Physical Review Letters, 98(15),pp. 150601, 2007.

[7] Daw, C. S., Finney, C. E. A. \& Kennel, M. B., Symbolic approach for measuring temporal "irreversibility". Physical Review E, 62(2),pp. 1912, 2000.

[8] Costa, M., Goldberger, A. L. \& Peng, C. K., Broken asymmetry of the human heartbeat: loss of time irreversibility in aging and disease. Physical Review Letters, 95(19),pp. 198102, 2005.

[9] Costa, M. D., Peng, C. K. \& Goldberger, A. L., Multiscale analysis of heart rate dynamics: entropy and time irreversibility measures. Cardiovascular Engineering, 8(2),pp. 88-93, 2008.

[10] Yao, W. P., Liu, T. B., Dai, J. F. \& Wang, J., Multiscale permutation entropy analysis of electroencephalogram. Acta Physica Sinica, 63(7),pp. 078704, 2014.

[11] Costa, M., Goldberger, A. L. \& Peng, C. K., Multiscale entropy analysis of complex physiologic time series. Physical Review Letters, 89(6),pp. 068102, 2002.

[12] Shen, W. \& Wang, J., Time irreversibility analysis of ECG based on symbolic relative entropy. Acta Physica Sinica, 60(11), 2011.

[13] Yang, X. D., Ning, X. B., He, A. J. \& Du, S. D., Mass exponent spectrum analysis of human ECG signals based on multiple scale factors. Acta Physica Sinica, 57(3),pp. 1514-1521, 2008.

[14] Wessel, N., Ziehmann, C., Kurths, J., Meyerfeldt, U., Schirdewan, A. \& Voss, A., Short-term forecasting of life-threatening cardiac arrhythmias based on symbolic dynamics and finite-time growth rates. Physical Review E, 61(1),pp. 733-739, 2000. 